\title{
Failure of the Fisher matrix when including tidal terms: Considering construction of template banks of tidally deformed binary neutron stars
}

\author{
Ian Harry $\odot$ and Andrew Lundgren \\ University of Portsmouth, Institute of Cosmology and Gravitation, Portsmouth PO1 3FX, United Kingdom
}

(Received 5 January 2021; accepted 13 July 2021; published 9 August 2021)

\begin{abstract}
Recent gravitational-wave observations have begun to constrain the internal physics of neutron stars. However, current detection searches for neutron star systems assume that potential neutron stars are lowmass black holes, ignoring any affect on the gravitational-wave signal due to the internal neutron star physics. We wish to create a template bank of binary neutron star waveforms including the effect of tidal deformability. However, we find that the Fisher matrix, which is commonly used to approximate match calculations when placing template banks, is unsuitable to predict the match between two binary neutron star waveforms. We find that the Fisher matrix can predict errors on the mismatch that are larger than $100 \%$ when attempting to identify waveforms with a match of 0.97 . We explore the regime in which the Fisher matrix cannot be trusted and examine why it breaks down. We demonstrate that including higher-order terms in the Taylor series expansion of the match can reliably compute matches for these examples, but that it is prohibitively computationally expensive to do so. Finally, we demonstrate that stochastic placement can still be used to construct a template bank of tidally deformed neutron star waveforms.
\end{abstract}

DOI: $10.1103 /$ PhysRevD.104.043008

\section{INTRODUCTION}

Second-generation gravitational-wave observatories Advanced LIGO [1] and Advanced Virgo [2] have recently opened the gravitational-wave Universe to us. Observations of binary black holes [3,4], binary neutron stars [5,6], and neutron star-black hole mergers [7] have demonstrated that a rich wealth of systems can be observed through gravitational waves. As these observatories continue to increase in sensitivity, and are joined by additional observatories in Japan [8] and India [9], we can continue to expect new discoveries and better understanding of the gravitationalwave Universe in the coming years.

The observation of compact binary mergers has required complex search algorithms [10-16]. These search algorithms rely on matched-filtering a large set of filter waveforms against the full set of data [10,11]. The filter waveforms are generated from models designed to create reliable waveforms for any input physical parameters $[17,18]$. These sets of filter waveforms, commonly referred to as a "template bank," are normally constructed such that any physical signal in the parameter space of interest would be found with no more than a fixed loss (normally 3\%) in signal-to-noise ratio [11].

There are two main methods that are used for creation of template banks. The first approach, stochastic placement, involves randomly choosing a very large set of points within the parameter space and then iterating through these points, accepting only points that are not "close" to any point already accepted [19-25]. The second approach, geometric placement, involves using the Fisher matrix to define a metric on the parameter space and then placing a lattice of points to cover the full space [26-34]. Stochastic placement offers the benefit of flexibility; it can be applied to any placement problem. Geometrical placement offers the benefit that it will place more efficient banks -in terms of fewer templates to achieve a given covering criterionbut it does require an underlying metric accurately describing the parameter space. Current wisdom states that geometric placement is the best option when placing template banks of binary neutron stars, when analytical metrics describing inspiral-only waveforms can be accurately applied. For heavier systems, where the merger is important, stochastic placement is commonly used. In addition, recent work has explored the development of hybrid methods attempting to combine the advantages of both methods to place efficient template banks $[35,36]$.

LIGO/Virgo searches for compact binary mergers have always searched for binary neutron star systems using waveforms that assume both bodies are point particles [11]. In reality, the internal composition of the neutron star can be important in the dynamics of the system. Tidal-induced deformation [37-41], spin-induced deformation [42,43], and the complex physics describing the postmerger behavior of a binary neutron star merger (see, e.g., Ref. [44]) can all leave observable imprints in the gravitational-wave signal. In terms of observing gravitational-wave signals from binary neutron star mergers, it has already been demonstrated that the tidal-induced deformation does have an impact on the observability of such systems [45]. This effect is not large enough to cause us to miss "loud" signals 
such as GW170817 or GW190425, but it can cause some reduction in sensitivity to signals close to the detection threshold [45]. From measurements of GW170817 and GW190425, we already have some indication of the equation of state governing the internal physics of neutron stars $[46,47]$. It is natural to include this knowledge in the construction of future template banks for LIGO and Virgo searches, and to place lattices of templates including the neutron stars' equation of state.

In this paper, we will aim to demonstrate how to place template banks of binary neutron star waveforms that include the effects of tidal-induced and spin-induced deformation. We begin by extending the current geometrical approaches to include the high-order tidal terms in the metric used. In doing so, we demonstrate the inadequacy of the Fisher matrix approximation for predicting matches between waveforms with tidal corrections modeled in the frequency domain using the post-Newtonian approximation. This result has implications not only in terms of searches, but also in parameter inference studies that use the Fisher matrix as a proxy for numerical match computations. We further explore the validity of the Fisher matrix in more general terms, and we show how including higherorder terms in the analytical Taylor series expansion of the match can improve the accuracy, but they are difficult to include for template placement. Finally, we demonstrate that stochastic methods are, as expected, capable of placing template banks of binary neutron stars including tidal terms, and we discuss the efficiency of such methods.

\section{THE FISHER MATRIX}

We begin by describing how the Fisher matrix is currently used to approximate the overlap between two waveforms in gravitational-wave astronomy. This formalism is widely used in the literature, but we include it here for completeness and for clarity when we later introduce higher-order corrections to the Taylor series expansion of the overlap. Specifically, we draw from the methods and formalism derived in Refs. [26-34,48] when formulating this section.

A common problem in gravitational-wave astronomy is determining the overlap between two waveforms. This is a measure of how well two waveforms agree with each other, and it can be used to assess how well we might observe a given signal if using a search template with different physical parameters as a filter. It can also be used to determine the chance that detector noise will make it impossible to distinguish two waveforms with different parameters. This overlap can be computed numerically as

$$
\left\langle h_{1} \mid h_{2}\right\rangle=4 \Re\left[\int_{0}^{\infty} d f \frac{\tilde{h}_{1}^{*}(f) \tilde{h}_{2}(f)}{S_{h}(f)}\right] .
$$

It is standard practice to normalize $h_{1}$ and $h_{2}$ such that $\left\langle h_{1} \mid h_{1}\right\rangle=1$ and $\left\langle h_{2} \mid h_{2}\right\rangle=1$. This implies that if $\left\langle h_{1} \mid h_{2}\right\rangle=1$, then these two waveforms are identical, whereas if $\left\langle h_{1} \mid h_{2}\right\rangle=0$, then the waveforms are completely "orthogonal" in parameter space. Another common measure directly related to the overlap is the "match," which is the overlap maximized over a phase and time shift between the two waveforms.

There are many purposes, of which template bank placement is one example, for which the match between two waveforms must be computed many times. This can computationally become very expensive [21]. In such cases, it is strongly desirable to be able to rapidly compute the match between any two waveforms. More specifically, we often want to compute the match between any two waveforms if it is near to 1 , or quickly identify that the match is not close to 1. Many optimizations exist for the numerical match code, used in the context of template bank placement [24,25] or for parameter inference [49-51].

Nevertheless, despite these optimizations, it is much more efficient if the overlap and match can be computed analytically. We define the overlap between two nearby waveforms, one with parameters denoted by $\theta_{i}$ and another with parameters denoted by $\theta_{i}+\Delta \theta_{i}$, as

$$
O\left(\theta_{i}, \Delta \theta_{i}\right)=\left\langle h\left(\theta_{i}\right) \mid h\left(\theta_{i}+\Delta \theta_{i}\right)\right\rangle .
$$

By definition $O\left(\theta_{i}, 0\right)=1$. If we are interested in computing this quantity in the limit that $\Delta \theta_{i}$ is small, then we can Taylor-expand:

$$
O\left(\theta_{i}, \Delta \theta_{i}\right)=O\left(\theta_{i}, 0\right)+\frac{\partial O}{\partial \theta^{i}} \Delta \theta_{i}+\frac{1}{2 !} \frac{\partial^{2} O}{\partial \theta^{i} \partial \theta^{j}} \Delta \theta_{i} \Delta \theta_{j}+\cdots
$$

$O\left(\theta_{i}, 0\right)$ must be a maximum, as it is not possible for the overlap to be larger than 1 ; therefore, $\frac{\partial O}{\partial \theta^{i}}$ must be 0 . Neglecting higher-order terms in the Taylor expansion, we can then write this as

$1-O\left(\theta_{i}, \Delta \theta_{i}\right) \approx-\frac{1}{2 !} \frac{\partial^{2} O}{\partial \theta^{i} \partial \theta^{j}} \Delta \theta_{i} \Delta \theta_{j} \equiv g_{i j} \Delta \theta_{i} \Delta \theta_{j}$.

In doing so, we can see that it is natural to define a metric

$$
g_{i j}=-\frac{1}{2 !} \frac{\partial^{2} O}{\partial \theta^{i} \partial \theta^{j}}
$$

on the parameter space, known as the Fisher matrix. Following Ref. [48], this is often equivalently written as

$$
g_{i j}=\frac{1}{2}\left\langle\frac{\partial h}{\partial \theta^{i}} \mid \frac{\partial h}{\partial \theta^{j}}\right\rangle
$$

however, the form in Eq. (5) will be more convenient for this work. The match can then be obtained from the Fisher matrix by projecting out the phase- and time-shift 
coordinates of $g_{i j}$ as described in Ref. [29]. In what follows, we will use the overlap exclusively, unless we specifically state otherwise.

Generically, the Fisher matrix here does have a dependence on $\theta_{i}$-the position in parameter space. However, if $g_{i j}$ can be evaluated quickly, the Fisher matrix offers a way to quickly evaluate the overlap between two waveforms that are "close" in parameter space.

\section{A. Fisher matrix and TaylorF2}

The "TaylorF2" waveform model [52,53], based on an analytical Fourier transform of the "TaylorT2" model $[53,54]$ via the stationary phase approximation, is a good candidate for use with the Fisher matrix. The TaylorF2 waveform model only models the inspiral part of a gravitational-wave signal, using the post-Newtonian expansion $[52,53]$. It can analytically be written as

$$
\tilde{h}(f)=A\left(f ; \mathcal{M}, D_{L}, \xi_{x}\right) e^{-i \Psi\left(f ; \lambda_{i, j}\right)} .
$$

The amplitude term here, $A$, depends on the chirp mass of the system $\mathcal{M}$, the luminosity distance $D_{L}$, and the orientation angles of the source with respect to the observer $\xi_{x}$. Most of this dependence is removed when normalizing the waveform. The important evolution here happens in the phase term, $\Psi$. This depends on the intrinsic masses and spins of the component objects via the $\lambda_{i, j}$ coefficients. The phase can be expressed in terms of these coefficients according to

$$
\Psi=2 \pi f t_{c}-\phi_{c}+\sum_{i} \sum_{j} \lambda_{i, j} f^{(i-5) / 3} \log ^{j} f,
$$

following the notation used in Ref. [34]. These $\lambda_{i, j}$ coefficients are the post-Newtonian coefficients of the waveform. These terms have quite complicated dependencies on the physical parameters and can be seen in their full form in, for example, Ref. [55]. The $i$ coefficient, divided by 2 , denotes what is commonly referred to when describing the post-Newtonian (PN) orders. For example, the $\lambda_{2,0}$ component denotes the " 1 PN" term. The $j$ component denotes the presence, and power, of any log terms in the coefficient.

We also note that the $\phi_{c}$ and $t_{c}$ terms, corresponding to the coalescence time and coalescence phase, can also be absorbed into the $\lambda_{i, j}$ coefficients by noting that a shift in $\phi_{c}$ will correspond to a shift in the $\lambda_{5,0}$ component, the $2.5 \mathrm{PN}$ term. Similarly, a shift in $t_{c}$ is equivalent to a shift in the $\lambda_{8,0}$ component, the $4 \mathrm{PN}$ term. These terms are then projected out if one wants to compute the match, instead of the overlap, between two waveforms.

This form is advantageous because, for TaylorF2, it allows one to easily compute the differential of $O\left(\theta, \Delta \theta_{i}\right)$. In Ref. [31], an illustration is given of how to compute this differential with respect to any physical parameter.
However, the forms given in Ref. [31] can be greatly simplified by using the fact that the $\lambda_{i, j}$ coefficients themselves present a natural coordinate system. Therefore, in this work, we compute the Fisher matrix in the $\lambda_{i, j}$ coordinate system. Using these coordinates, it can be shown that

$$
\frac{\partial^{2} O}{\partial \lambda_{i, j} \partial \lambda_{k, l}}=-4 \Re\left[\int_{f_{L}}^{f_{U}} d f \frac{f^{(i+k-17) / 3} \log ^{j+l} f}{S_{h}(f)}\right],
$$

where $f_{U}$ and $f_{L}$ are the upper and lower limits to the integration.

In the $\lambda_{i, j}$ coordinate system, we can clearly see that the Fisher matrix does not depend on the position in the parameter space; it is globally flat. One can take this further, orthonormalizing this parameter space and identifying principal directions. This then offers a natural system in which to place a reduced-dimension lattice of points to form a template bank [33] using standard techniques for sphere packing in Cartesian coordinate systems [56].

In doing this, a number of approximations are made, and it is important to clearly state these again before continuing. First, when using the TaylorF2 waveform model, we implicitly assume that it is a fully accurate representation of the emitted gravitational-wave signal. TaylorF2 does not include the merger and ringdown components of the signal, so it will not accurately reproduce the final stages of a binary neutron star merger. We will not explore this feature in this work, as TaylorF2 is the only waveform for which a globally flat metric has been defined, and the validity of TaylorF2 has been extensively studied elsewhere (e.g., Ref. [55]). We emphasize that we are in no way dismissing this effect; accurately modeling such waveforms is of paramount importance. However, we wish to explore in detail the other approximations which are normally ignored in the literature. TaylorF2 is also used extensively in template bank placement, detection searches, and parameter estimation studies of binary neutron systems. The second approximation made is that the TaylorF2 model has some specific start and end frequencies, outside of which the frequency content is 0 . To achieve a globally flat metric, one must also assume that these limits are the same for any value of the physical parameters of the system. The lower frequency limit is normally chosen through practical constraints, either corresponding to a point at which the power spectral density increases rapidly, or limiting the length of the filters used in the search. The upper frequency cutoff is normally chosen to take some value, between 1000 and $2000 \mathrm{~Hz}$, roughly corresponding to the innermost stable circular orbit of a binary neutron star system where both components have a mass of $1.4 M_{\odot}$. The power contribution above $1000 \mathrm{~Hz}$ is small for such systems, and so the exact value chosen here, and the fact that it will change with mass, does not matter much. For example, for a nonspinning equal-mass binary neutron star system with 
components of $1.4 M_{\odot}, 99.7 \%$ of the signal power is emitted in gravitational waves below $1000 \mathrm{~Hz}$ (assuming the TaylorF2 model). The third assumption is that terms beyond the Fisher matrix term in the Taylor series expansion of the match are negligible. In this work, our focus will be on exploring the validity of assuming that the higherorder terms in the Taylor expansion are negligible, in particular for binary neutron star systems. We also explore the consequences of assuming a constant frequency cutoff. It is important to highlight that our results are specific to the TaylorF2 waveform, and the results we find may differ if other waveform models are utilized.

\section{NEUTRON STARS ARE NOT BLACK HOLES}

There a number of key differences in the gravitationalwave signal emitted by a binary neutron star system and that emitted by a binary black hole system with the same component masses and spins. These differences arise due to the internal composition of the neutron star and encode information that can let us study the behavior of matter in one of the most extreme environments in the Universe.

First, the postmerger phase of a binary neutron star system will be rich with physics (see Ref. [57] for a brief summary). Will a short-lived hypermassive neutron star be formed, emitting gravitational radiation before collapsing to a black hole [58-61]? Being able to observe the postmerger phase of such a system will offer valuable insights into the internal physics of neutron stars [62-65]. However, such signatures will be emitted at high frequencies, which are very challenging for current observatories to detect (see Sec. II in Ref. [66] for a detailed discussion). Sensitivity to such signatures is a key science objective for proposed third-generation observatories, such as the Einstein Telescope and Cosmic Explorer [67-69]. In this work, we neglect such effects. The TaylorF2 waveform does not include any merger or postmerger phase, and such effects should not be important until third-generation observatories are built [66].

A second effect occurs due to the spherical asymmetry of rotating neutron stars $[42,43]$. This effect adds terms to the post-Newtonian expansion, beginning at the $2 \mathrm{PN}$ order (the $\lambda_{4,0}$ term in our notation above). It has been found that while this term can have a significant effect on the emitted waveform, it is strongly degenerate with the spins of the bodies themselves and does not allow direct measurements of nuclear physics [70].

The most important effect for us to consider is the deformation of the neutron stars due to tidal interactions [37-41]. This tidal deformation occurs late in the evolution of the system-very near to the merger-with leading terms at $5 \mathrm{PN}$ and $6 \mathrm{PN}$ order $\left(\lambda_{10,0}\right.$ and $\left.\lambda_{12,0}\right)$. As these terms enter the expansion very differently than other terms, they are largely orthogonal, allowing the tidal deformability to be measured [71]. Therefore, it is natural to want to include the effect of these terms in the bank of templates used for observing binary neutron stars. Studies in Refs. $[45,70]$ have shown that one incurs a non-negligible loss in sensitivity if these terms are ignored in detection searches.

\section{USING THE FISHER MATRIX TO PREDICT OVERLAPS WITH TIDAL TERMS}

In this section, we begin by exploring the validity of using existing geometric template bank placement methods to place a template bank of neutron star systems including the deformation due to tidal interactions and the deformation due to the bodies' own rotation.

The method described in Ref. [34] already includes the formalism needed to include the deformability due to the bodies' own rotation. This is simply included by adding the effect to the existing post-Newtonian orders with a suitable range of values encompassing the uncertainty in modeling the neutron star's internal physics. Similarly, the tidal deformability can also be included by introducing the $5 \mathrm{PN}$ and $6 \mathrm{PN}$ terms in the existing set of parameters being considered. One then allows a suitable range of values, again given by some range of possible equations of state, and proceeds using the methods described in Ref. [34].

However, for this approach to work well, the Fisher matrix approximation must be able to predict well the overlaps between two waveforms that vary in the $5 \mathrm{PN}$ and 6 PN terms $\left(\lambda_{10,0}\right.$ and $\left.\lambda_{12,0}\right)$ corresponding to changes in the tidal deformation parameters. To check if this is the case, we generate two TaylorF2 waveforms and add a small perturbation in the $5 \mathrm{PN}$ and $6 \mathrm{PN}$ terms to one of them before computing the overlap. By numerically varying the ratio of the perturbation in the two terms, and the magnitude of the deviation, we can identify the line of perturbations that produce a specific overlap. We then use the Fisher matrix to predict the perturbations needed to produce the same overlap and can then compare the agreement between the predicted Fisher matrix and the numerical value. In these overlap calculations, we use a lower frequency cutoff of $15 \mathrm{~Hz}$ and an upper frequency cutoff of $2048 \mathrm{~Hz}$. The waveform model is generated at all values between these limits even if the innermost stable circular orbit occurs below $2048 \mathrm{~Hz}$.

We plot the results of doing this for an overlap of 0.97 in Fig. 1. One can clearly see that the Fisher matrix is very poorly predicting the overlap between these two waveforms. For comparison, in Fig. 2 we produce the same plot, except that we perturb the $0 \mathrm{PN}$ and $1 \mathrm{PN}$ terms $\left(\lambda_{0,0}\right.$ and $\left.\lambda_{2,0}\right)$. In this case, the Fisher matrix predicts the overlap well. We also show in Fig. 2 the result of perturbing the physical chirp mass $(\mathcal{M})$ and symmetric mass ratio $(\eta)$ terms. Perturbing these terms will perturb all PN terms up to the 3.5 PN order, but the dominant effect will be changes in the leading-order PN terms. The Fisher matrix also predicts the overlap well in this case. 


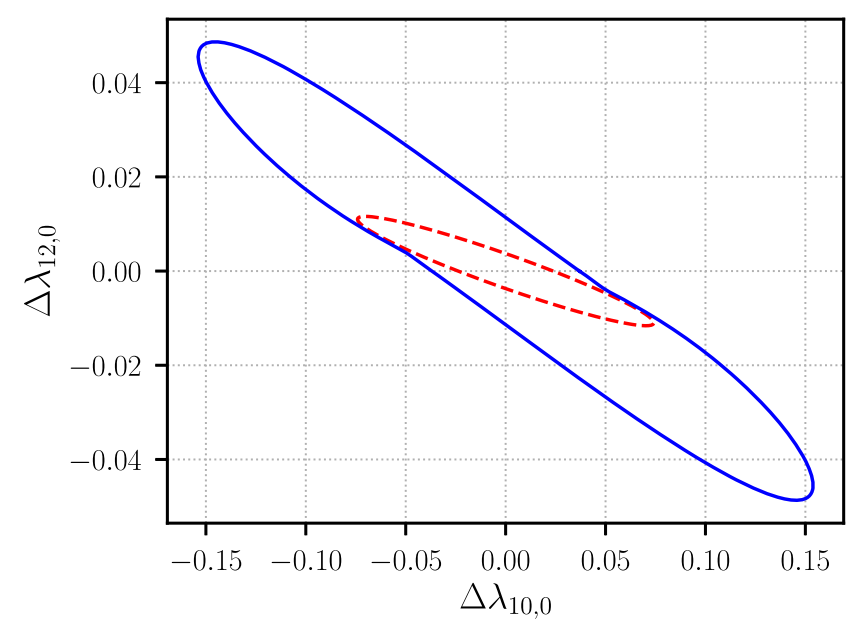

FIG. 1. The perturbation that needs to be added to the $5 \mathrm{PN}$ and $6 \mathrm{PN}$ terms to achieve an overlap of 0.97 with an unperturbed TaylorF2 waveform. The blue solid line shows the numerical overlap. The red dashed line shows the overlap predicted by the Fisher matrix. For an animated version showing more detail, please see the Supplemental Material [72].

It is clear from these plots that the Fisher matrix can predict well overlaps that are 0.97 or larger when we are changing the dominant $0 \mathrm{PN}$ - and $1 \mathrm{PN}$-order terms. It also predicts the overlap very badly when changing the 5 PN and $6 \mathrm{PN}$ terms where tidal deformability is first evident. We will now proceed to try to understand at what PN orders the Fisher matrix performs well, and where it does not. We will also try to understand why it works well at some orders, and poorly at others.

\section{A. Where does the Fisher matrix fail?}

We have demonstrated that the Fisher matrix fails to correctly predict overlaps when one is varying tidal terms. This, in turn, will render the placement algorithm described in Ref. [34] ineffectual for placing template banks of binary neutron star systems with tidal deformations. However, it is informative to ask where in the PN expansion the Fisher matrix approximation breaks down and to see if we can understand the conditions under which the Fisher matrix breaks down.

We consider the following: For each of the terms in the post-Newtonian expansion, we vary the value of that term, computing overlaps numerically, until we empirically determine the change needed to produce a specified match (e.g., 0.97) if only that term is changing. We can then compute the overlap that the Fisher matrix predicts for this change and compare the two. This can be applied to all terms in the expansion; for example, while there is no $0.5 \mathrm{PN}$ term predicted by general relativity, we can still shift the TaylorF2 waveform phasing as if this term were present to determine the mismatch due to its variation. We do this for all linear-order terms between $0 \mathrm{PN}$ and $6 \mathrm{PN}\left(\lambda_{0,0}\right.$ to $\lambda_{12,0}$ in our notation) and for all log terms between $0 \mathrm{PN}$ and
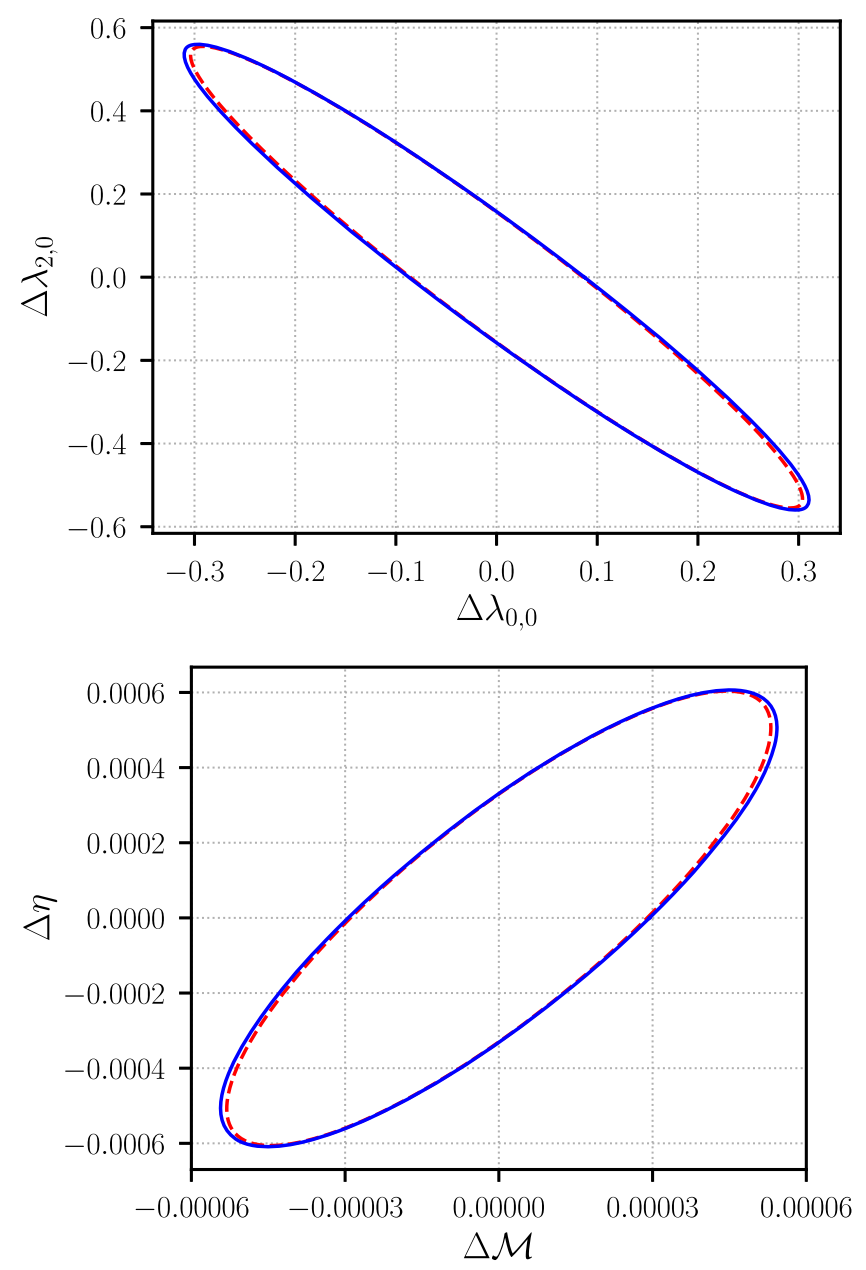

FIG. 2. The perturbation that needs to added to the $0 \mathrm{PN}$ and 1 PN terms (top) or $\mathcal{M}$ and $\eta$ terms (bottom) to achieve a overlap of 0.97 with an unperturbed TaylorF2 waveform. The blue solid line shows the numerical overlap. The red dashed line shows the overlap predicted by the Fisher matrix. For an animated version showing more detail, please see the Supplemental Material [72].

$6 \mathrm{PN}\left(\lambda_{0,1}\right.$ to $\lambda_{12,1}$ in our notation). This is shown in Fig. 3. For completeness, the "fractional error in overlap" quoted in these plots is defined as

$$
\frac{(1-N)-(1-A)}{(1-N)}
$$

where $N$ denotes the numerical overlap and $A$ denotes the analytical prediction, in this case computed using the Fisher matrix.

From Fig. 3, we can see that the Fisher matrix appears to be most reliable when computing overlaps at a postNewtonian order of 2.5 and diverges on either side of this. For larger post-Newtonian orders, this divergence happens quickly, and we see again that at post-Newtonian orders associated with tidal effects, the Fisher matrix is not predicting the overlap accurately considering waveforms 

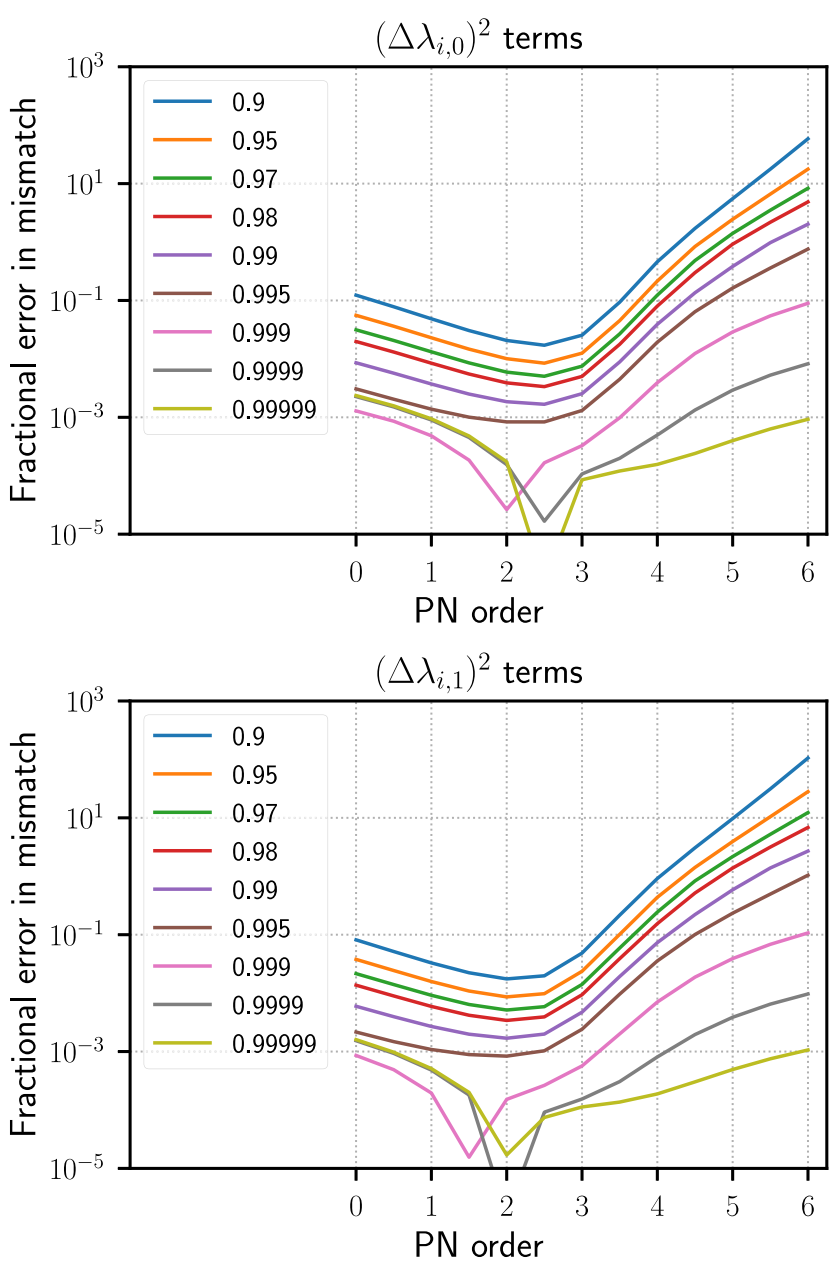

FIG. 3. The accuracy at which the Fisher matrix predicts the overlap when considering two waveforms that differ by a change in a single $\lambda_{i, j}$ term in the post-Newtonian expansion. Shown for $\lambda_{i, 0}$ (top) and $\lambda_{i, 1}$ (bottom).

with a numerical overlap of 0.97 . We notice that when using overlaps very close to 1 , the Fisher matrix does predict the overlaps well, but there does appear to be an error of roughly $0.1 \%$ at all post-Newtonian orders except 2.5. We have not been able to identify where this error comes from-we suspect some subtle systematic effectbut it does not affect our conclusions.

\section{B. The importance of beyond-leading-order terms in the match calculation}

We identified previously the assumptions that are made when defining the Fisher matrix approximation of the overlap. Now we investigate the validity of the assumption that terms beyond the leading-order term in the Taylor series expansion of the overlap are negligible. We note that there has been some exploration of the importance of these terms in previous works [73-77]. However, these works differ from this one, in that they focus on the bias that neglecting such terms would have on inferring the parameters of gravitational-wave signals, and they do not consider the specific problem of tidal terms. If we go back to Eq. (3) and expand the match to fourth order in $\Delta \theta_{i}$, we can write the match as

$$
1-M\left(\theta_{i}, \Delta \theta_{i}\right)=-\frac{1}{2 !} \frac{\partial^{2} M}{\partial \theta^{i} \partial \theta^{j}} \Delta \theta_{i} \Delta \theta_{j}-P_{4},
$$

where $P_{4}$ is the fourth-order correction term given by

$$
P_{4}=\frac{1}{4 !} \frac{\partial^{4} M}{\partial \theta^{i} \partial \theta^{j} \partial \theta^{k} \partial \theta^{l}} \Delta \theta_{i} \Delta \theta_{j} \Delta \theta_{k} \Delta \theta_{l} .
$$

In this case, the $P_{4}$ term involves a rank-4 tensor. ${ }^{1}$ If we consider that a standard TaylorF2 waveform might contain contributions from $O(10)$ terms, this tensor must contain $10^{4}$ terms. As with the Fisher matrix, there is a lot of symmetry in this tensor, and many terms are repeated. Our use of $\lambda_{i, j}$ coordinates is again useful here if we try to evaluate this rank-4 tensor. In particular, we can show that

$$
\begin{aligned}
& \frac{\partial^{4} M}{\partial \lambda_{i, j} \partial \lambda_{k, l} \partial \lambda_{m, n} \partial \lambda_{o, p}} \\
& \quad=-4 \Re\left[\int_{f_{L}}^{f_{U}} d f \frac{f^{(i+k+m+o-27) / 3} \log ^{j+l+n+p} f}{S_{h}(f)}\right] .
\end{aligned}
$$

In this way, it is also easy to expand this to even higherorder terms in the Taylor series expansion.

To investigate the importance of these terms, we again follow our procedure in Fig. 3 where we only perturb one of the PN terms and compute the resulting overlap. The advantage to doing this is that there is still only one term in the rank-4 tensor that is nonzero, and indeed only one term in all the higher-order tensors as well, allowing us to compute analytical overlap to very high order in the Taylor expansion when varying only a single PN order.

As with Fig. 3, we numerically determine the perturbation needed to produce a numerical overlap of a given value. We then compute the analytical overlap up to various orders in the Taylor expansion. In Fig. 4, we show results at fourth order, 20th order, and 40th order in the Taylor expansion. A short animation, which can be viewed in the Supplemental Material [72], perhaps shows this better, demonstrating how the agreement evolves as we incrementally add increasingly higher-order terms.

We notice that terms at 2.5 PN order and below are in most cases not highly sensitive to the inclusion of the higher-order terms. Adding the fourth-order term does improve the accuracy of the computation, which is especially noticeable at smaller values of the numerical overlap. The predicted overlap quickly converges, though. It still does not converge to an error of 0 , hinting again at the

\footnotetext{
${ }^{1}$ Here we use the term "tensor" as it is used in computational science, rather than in mathematics. Formally, this is not a tensor, as it will not obey the coordinate transformation rules.
} 

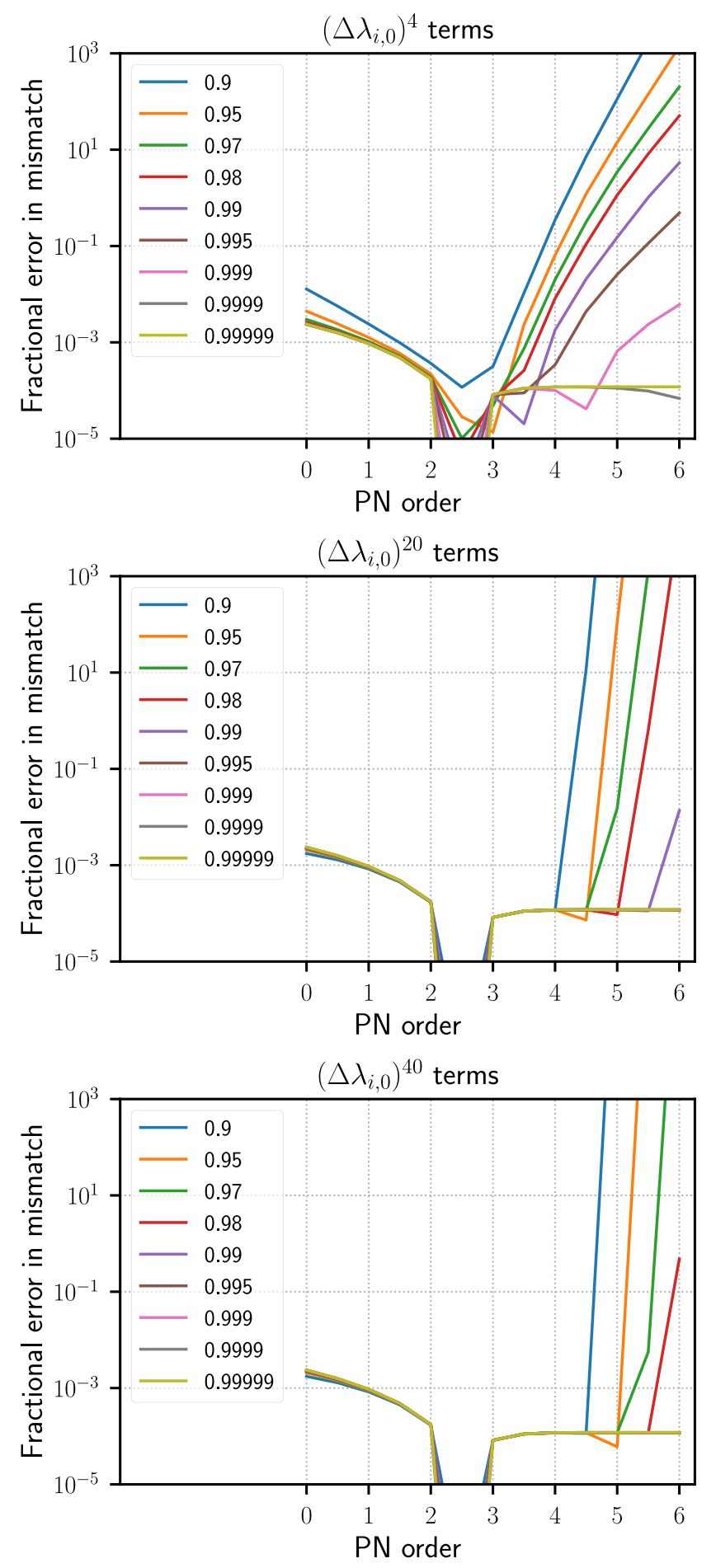

FIG. 4. The accuracy at which the analytical approximation predicts the overlap between two waveforms that differ by a change in a single $\lambda_{i, 0}$ term in the post-Newtonian expansion. Shown for $\lambda_{i, 0}$ terms with corrections up to 4 th (top), 20th (middle), and 40th (bottom) order in the Taylor series expansion of the overlap. Note that the Fisher matrix is the second-order correction, and all odd-ordered terms are 0 . An animation of this figure showing the inclusion of higher-order corrections one by one can be found in the Supplemental Material [72]. presence of some subtle systematic between the Fisher matrix and the numerical overlap that we have not accounted for.

At PN orders above 2.5 PN, we observe quite different behavior. It is often the case that the addition of higherorder terms first causes the accuracy of the overlap calculation to decrease (in many cases predicting overlaps much greater than 1 , or much less than 0 , which of course are not possible physically, but are possible from this analytical prediction). However, as increasingly higherorder terms are added, the analytical overlap does converge to be consistent with the numerical value. For the $6 \mathrm{PN}$ term at an overlap of 0.97 , this required adding terms up to 60th order in the Taylor series expansion. This indicates that the unreliability of the Fisher matrix predictions is due to the missing higher-order terms in the expansion of the analytical overlap computation.

\section{What is special about the 2.5 PN term}

Our results in Fig. 4 beg the question of why the Fisher matrix performs so poorly for the large post-Newtonian terms and why the 2.5 post-Newtonian term is the one most easily predicted. To understand this, we examine the generic form of the rank- $N$ tensor that would represent the $N$ th-order term in the Taylor expansion. Considering only the non-log terms $\lambda_{i, 0}$, this can be generically written as

$\frac{\partial^{2} M}{\partial \lambda_{i} \partial \lambda_{j} \partial \lambda_{k} \cdots}=4 \Re\left[(-1)^{N / 2} \int_{0}^{\infty} d f \frac{f^{(i+j+k+\cdots-5 N-7) / 3}}{S_{h}(f)}\right]$.

From examination of this equation, we can see that the power in the numerator of the integral is equal to $(i+j+k+\cdots-$ $5 N-7) / 3$ or $((i-5)+(j-5)+(k-5)+\cdots-7) / 3$. Therefore, if the sum of the various terms (after subtracting 5 from each) is larger than 7, the power in the numerator of the integral is positive. At high frequencies, $S_{h}(f)$ is dominated by the shot noise, which is proportional to $f^{2}$. Therefore, there will be cases where the power in the numerator is larger than the $f^{2}$ term in the denominator, which will make this calculation very sensitive to content at high frequencies. It also makes the calculation very sensitive to the choice of the upper frequency cutoff, which we will explore further in the next subsection. Even for the Fisher matrix, changes in the $6 \mathrm{PN}$ term result in a numerator term proportional to $f^{7 / 3}$, which is rising faster than the shot noise contribution in the denominator.

If one considers the fourth-order (and higher) terms in the Taylor series expansion, the power of the numerator can become very large. Therefore, when we move to a higher order, while the $\Delta \theta_{i}$ terms and the inverse factorial term will become increasing small, the integral will become increasingly large. In contrast, at $2.5 \mathrm{PN}$ order, the numerator 
of the integral remains $f^{-7 / 3}$ regardless of what order in the Taylor expansion we are considering. In this case, the higherorder terms in the expansion will quickly be negligible. Finally, at low PN orders the numerator will have a negative power, which will also grow increasingly large for higher terms in the Taylor series expansion. Here also, these terms can rise faster than the noise floor at low frequencies, although this is somewhat limited by the practical need to start the integral from some low-frequency cutoff.

\section{The practicality of including higher-order terms in the match approximation}

We have demonstrated that the Fisher matrix poorly predicts the overlap between nearby waveforms when varying terms at high-post-Newtonian order, as is necessary when considering changes in the tidal deformation terms. We have also demonstrated that we can accurately predict such overlaps analytically by including higher-order corrections to the Fisher matrix. However, in our tests we simplified the computation of these higher-order terms by changing only a single post-Newtonian term at a time.

It is much more computationally impractical to compute these higher-order terms analytically when varying multiple post-Newtonian terms-for example, if changing the masses of a binary neutron star waveform with a given equation of state. The reason for this can be seen if we again consider Eq. (11). Here we include the fourth-order $P_{4}$ term, which might include $\sim 10^{4}$ terms for a standard TaylorF2 waveform. Even after removing duplicated terms due to symmetry, we can see that if we include higher-order terms in the analytical computational of the overlap, the computational complexity will grow exponentially with additional terms. If we include terms to 20th order, which still does not accurately predict matches for tidal postNewtonian orders, we would require a rank-20 tensor with $\sim 10^{20}$ terms. It is simply not practical to compute the analytical match for waveforms including tidal corrections at sufficient order for the match to be reliable.

\section{E. Sensitivity of the analytical overlap to the upper frequency cutoff}

In the previous section, we have suggested that the validity of the Fisher matrix approximation for overlap computation is sensitive to the choice of the upper frequency cutoff. This is due to the fact that the numerator of the Fisher matrix integral for tidal terms can be as large as, or larger than, the shot noise term from the PSD in the denominator. We do expect the numerical overlap for TaylorF2 waveforms (when the waveform model does not include a termination condition) to be sensitive to the choice of upper frequency cutoff, but it is interesting to explore how both the numerical and

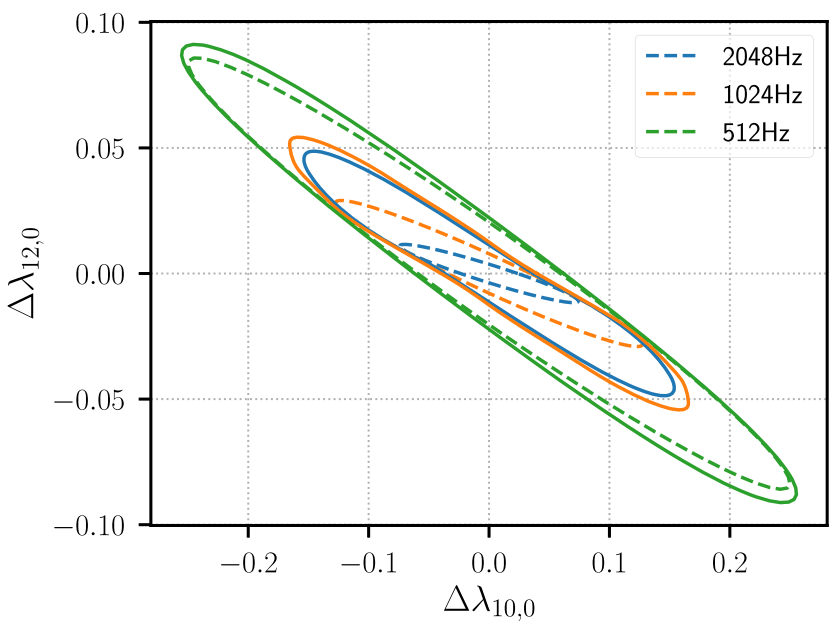

FIG. 5. The perturbation that needs to be added to the $5 \mathrm{PN}$ and $6 \mathrm{PN}$ terms to achieve a overlap of 0.97 with an unperturbed TaylorF2 waveform as a function of termination frequency. Solid lines show the numerical overlap. Dotted lines show the overlap predicted by the Fisher matrix. An animated version showing how this figure changes as a function of the termination frequency and as a function of the overlap can be found in the Supplemental Material [72].

Fisher-matrix-approximated overlap computations vary based on the choice of termination frequency.

To illustrate this, we reproduce Fig. 1, where we identified the perturbation in the $5 \mathrm{PN}$ and $6 \mathrm{PN}$ terms that is necessary to give an overlap of 0.97 with an unperturbed waveform. We compute this, as before, for both numerical and analytical overlap computations, but now compute this at three values of the termination frequency: $512 \mathrm{~Hz}, 1024 \mathrm{~Hz}$, and $2048 \mathrm{~Hz}$. The results of this are shown in Fig. 5.

From this, we observe the dependence of the numerical overlap of the TaylorF2 waveform on this choice. There is a significant difference between $512 \mathrm{~Hz}$ and $1024 \mathrm{~Hz}$, but not much of a change when increasing further to $2048 \mathrm{~Hz}$. In contrast, the accuracy of the Fisher matrix prediction is relatively good at $512 \mathrm{~Hz}$, but it decreases rapidly as the termination frequency continues to increase.

\section{F. Discussion}

The main result of this section is that the Fisher matrixderived metric is not suitable for predicting overlaps between two waveforms modeled by the analytical post-Newtonian TaylorF2 approximation. The approximation where the parameter space can be described by a Riemannian metric given by the Fisher matrix breaks down when considering TaylorF2 waveforms including tidal terms. We have demonstrated under which conditions one would obtain inaccurate overlaps and shown that including higher-order terms in the expansion of the expression for the overlap does resolve 
the problem, but it is computationally impractical for realistic use cases.

While we can look to mitigate this issue by carefully choosing the upper frequency cutoff to use, it is possible that this effect is particular to the waveform approximation used. $^{2}$ In particular, the TaylorF2 waveform is modeled as a series of post-Newtonian terms, dependent on increasing powers of frequency, that cut off abruptly at some termination condition. As discussed above, this can lead the derivatives used in the analytical overlap computation to be highly sensitive to the choice of termination frequency. It is possible that complete waveforms including inspiral, merger, and ringdown, which do not terminate abruptly, will behave better in this regard. However, there does not exist a parametrization for such waveforms that will allow us to construct a globally flat metric on the parameter space, which is required for many of the studies we perform here. Therefore, we leave an exploration of these effects with alternative waveform models for future work.

\section{USING STOCHASTIC PLACEMENT TO CREATE A TIDAL TEMPLATE BANK}

The focus of this work has been on exploring the inadequacies of the Fisher matrix approximation in accurately predicting the overlap between two waveforms due to differences in the tidal PN terms. However, the original motivation for exploring this was the goal of being able to create template banks of filter waveforms that include tidal corrections. We have already argued that geometric placement will not be appropriate here, which will also render the hybrid method proposed in Refs. [35,36] inappropriate here as well. However, stochastic template placement [19-25] can be used when computing overlaps numerically and therefore remains an appropriate solution to this problem.

To demonstrate this, we create a template bank of waveforms to cover systems with both component masses $\in[1,3] M_{\odot}$, with both component dimensionless spin magnitudes $\in[-0.05,0.05]$, and with the tidal deformability $\lambda$ parameter [38] for both bodies $\in[0,2000]$. We also assume the Advanced LIGO zero-detuned, high-power noise curve [1]. This bank contains 69250 templates, compared to a template bank generated in the same manner while assuming the tidal deformability for both bodies is 0 , which has 41439 templates. For comparison, a template bank generated with the geometric algorithm for the same

\footnotetext{
${ }^{2}$ We acknowledge the anonymous referee for their suggestion to explicitly include a paragraph of this nature.
}

parameter space, ignoring tidal deformability, contains 37977 templates.

We acknowledge that this parameter space is not particularly well motivated physically. Even if neutron stars with masses up to $3 M_{\odot}$ are possible, the tidal deformability parameter would be much smaller for such high-mass stars [47]. We can also reasonably assume that both neutron stars must be governed by the same underlying physics, such that there must be a tight correlation between the two stars' values of $\lambda$ given both masses. Including this physics would considerably reduce the number of additional templates required to create a tidal template bank. The techniques already exist to do this, as used in [78] for the case of targeting compact binary mergers that might power GRBs. We leave the choice of what exact constraints to use here open, as these constraints will likely evolve rapidly with future observations of binary neutron star mergers.

\section{CONCLUSION}

In this paper, we have explored the validity of the Fisher matrix for predicting waveform overlaps, with a particular focus on applications to template bank placement. We have found that the accuracy of the Fisher-matrix-predicted overlaps is poor when considering changes in postNewtonian terms larger than 4 PN order. We have investigated the reason for these poor predictions and identify that the neglected higher-order terms in the Taylor series expansion of the overlap are crucial for accurately predicting overlaps at high post-Newtonian order when using the TaylorF2 waveform model. Unfortunately, the computational cost of including such higher-order terms in analytic predictions quickly becomes prohibitive. (Indeed, it quickly becomes more expensive than a brute-force computation of the overlap.) We therefore conclude that the current geometric template bank placement algorithms are not suitable for placing template banks of binary neutron mergers including tidal deformability modeled using the TaylorF2 waveforms. We recommend that stochastic placement, using numerical computation of the match, be used instead.

\section{ACKNOWLEDGMENTS}

The authors would like to thank the anonymous referees for helpful feedback and critique on the first version of this manuscript. The authors would like to thank Bhooshan Gadre for useful discussion, comments, and feedback on this work. I. H. thanks the STFC for support through Grant No. ST/T000333/1. A. L. thanks the STFC for support through Grant No. ST/S000550/1. 
[1] J. Aasi et al. (LIGO Scientific Collaboration), Advanced LIGO, Classical Quant. Grav. 32, 074001 (2015).

[2] F. Acernese et al. (VIRGO Collaboration), Advanced Virgo: A second-generation interferometric gravitational wave detector, Classical Quant. Grav. 32, 024001 (2015).

[3] B. Abbott et al. (LIGO Scientific and Virgo Collaborations), Observation of Gravitational Waves from a Binary Black Hole Merger, Phys. Rev. Lett. 116, 061102 (2016).

[4] B. Abbott et al. (LIGO Scientific and Virgo Collaborations), GWTC-1: A Gravitational-Wave Transient Catalog of Compact Binary Mergers Observed by LIGO and Virgo during the First and Second Observing Runs, Phys. Rev. X 9, 031040 (2019).

[5] B. Abbott et al. (LIGO Scientific and Virgo Collaborations), GW170817: Observation of Gravitational Waves from a Binary Neutron Star Inspiral, Phys. Rev. Lett. 119, 161101 (2017).

[6] B. Abbott et al. (LIGO Scientific and Virgo Collaborations), GW190425: Observation of a compact binary coalescence with total mass $\sim 3.4 M_{\odot}$, Astrophys. J. Lett. 892, L3 (2020).

[7] R. Abbott et al. (LIGO Scientific and Virgo Collaborations), GW190814: Gravitational waves from the coalescence of a 23 solar mass black hole with a 2.6 solar mass compact object, Astrophys. J. Lett. 896, L44 (2020).

[8] T. Akutsu et al. (KAGRA Collaboration), Overview of KAGRA: Detector design and construction history, arXiv: 2005.05574.

[9] B. Iyer et al., Ligo-india (2011), https://dcc.ligo.org/ligoM1100296/public.

[10] B Allen, W. G. Anderson, P. R. Brady, D. A. Brown, and J. D. Creighton, FINDCHIRP: An algorithm for detection of gravitational waves from inspiraling compact binaries, Phys. Rev. D 85, 122006 (2012).

[11] S. Babak et al., Searching for gravitational waves from binary coalescence, Phys. Rev. D 87, 024033 (2013).

[12] S. Hooper, S. K. Chung, J. Luan, D. Blair, Y. Chen, and L. Wen, Summed parallel infinite impulse response (SPIIR) filters for low-latency gravitational wave detection, Phys. Rev. D 86, 024012 (2012).

[13] S. A. Usman et al., The PyCBC search for gravitational waves from compact binary coalescence, Classical Quant. Grav. 33, 215004 (2016).

[14] T. Adams, D. Buskulic, V. Germain, G. Guidi, F. Marion, M. Montani, B. Mours, F. Piergiovanni, and G. Wang, Lowlatency analysis pipeline for compact binary coalescences in the advanced gravitational wave detector era, Classical Quant. Grav. 33, 175012 (2016).

[15] C. Messick et al., Analysis framework for the prompt discovery of compact binary mergers in gravitational-wave data, Phys. Rev. D 95, 042001 (2017).

[16] T. Venumadhav, B. Zackay, J. Roulet, L. Dai, and M. Zaldarriaga, New search pipeline for compact binary mergers: Results for binary black holes in the first observing run of Advanced LIGO, Phys. Rev. D 100, 023011 (2019).

[17] A. Bohé et al., Improved effective-one-body model of spinning, nonprecessing binary black holes for the era of gravitational-wave astrophysics with advanced detectors, Phys. Rev. D 95, 044028 (2017).

[18] S. Khan, S. Husa, M. Hannam, F. Ohme, M. Pürrer, X. J. Forteza, and A. Bohé, Frequency-domain gravitational waves from nonprecessing black-hole binaries. II. A phenomenological model for the advanced detector era, Phys. Rev. D 93, 044007 (2016).

[19] I. Harry, S. Fairhurst, and B. Sathyaprakash, A hierarchical search for gravitational waves from supermassive black hole binary mergers, Classical Quant. Grav. 25, 184027 (2008).

[20] S. Babak, Building a stochastic template bank for detecting massive black hole binaries, Classical Quant. Grav. 25, 195011 (2008).

[21] I. W. Harry, B. Allen, and B. Sathyaprakash, A stochastic template placement algorithm for gravitational wave data analysis, Phys. Rev. D 80, 104014 (2009).

[22] G. M. Manca and M. Vallisneri, Cover art: Issues in the metric-guided and metric-less placement of random and stochastic template banks, Phys. Rev. D 81, 024004 (2010).

[23] P. Ajith, N. Fotopoulos, S. Privitera, A. Neunzert, and A. Weinstein, Effectual template bank for the detection of gravitational waves from inspiralling compact binaries with generic spins, Phys. Rev. D 89, 084041 (2014).

[24] S. Privitera, S. R. Mohapatra, P. Ajith, K. Cannon, N. Fotopoulos, M. A. Frei, C. Hanna, A. J. Weinstein, and J. T. Whelan, Improving the sensitivity of a search for coalescing binary black holes with nonprecessing spins in gravitational wave data, Phys. Rev. D 89, 024003 (2014).

[25] C. Capano, I. Harry, S. Privitera, and A. Buonanno, Implementing a search for gravitational waves from binary black holes with nonprecessing spin, Phys. Rev. D 93, 124007 (2016).

[26] B. Sathyaprakash and S. Dhurandhar, Choice of filters for the detection of gravitational waves from coalescing binaries, Phys. Rev. D 44, 3819 (1991).

[27] E. Poisson and C.M. Will, Gravitational waves from inspiraling compact binaries: Parameter estimation using second post-Newtonian wave forms, Phys. Rev. D 52, 848 (1995).

[28] R. Balasubramanian, B. Sathyaprakash, and S. Dhurandhar, Gravitational waves from coalescing binaries: Detection strategies and Monte Carlo estimation of parameters, Phys. Rev. D 53, 3033 (1996); Erratum, Phys. Rev. D 54, 1860 (1996).

[29] B. J. Owen, Search templates for gravitational waves from inspiraling binaries: Choice of template spacing, Phys. Rev. D 53, 6749 (1996).

[30] B. J. Owen and B. Sathyaprakash, Matched filtering of gravitational waves from inspiraling compact binaries: Computational cost and template placement, Phys. Rev. D 60, 022002 (1999).

[31] S. Babak, R. Balasubramanian, D. Churches, T. Cokelaer, and B. Sathyaprakash, A template bank to search for gravitational waves from inspiralling compact binaries: I. Physical models, Classical Quant. Grav. 23, 5477 (2006).

[32] T. Cokelaer, Gravitational waves from inspiralling compact binaries: Hexagonal template placement and its efficiency in detecting physical signals, Phys. Rev. D 76, 102004 (2007).

[33] D. A. Brown, I. Harry, A. Lundgren, and A. H. Nitz, Detecting binary neutron star systems with spin in advanced gravitational-wave detectors, Phys. Rev. D 86, 084017 (2012).

[34] I. W. Harry, A. H. Nitz, D. A. Brown, A. P. Lundgren, E. Ochsner, and D. Keppel, Investigating the effect of 
precession on searches for neutron-star-black-hole binaries with Advanced LIGO, Phys. Rev. D 89, 024010 (2014).

[35] S. Roy, A. S. Sengupta, and N. Thakor, Hybrid geometricrandom template-placement algorithm for gravitational wave searches from compact binary coalescences, Phys. Rev. D 95, 104045 (2017).

[36] S. Roy, A. S. Sengupta, and P. Ajith, Effectual template banks for upcoming compact binary searches in AdvancedLIGO and Virgo data, Phys. Rev. D 99, 024048 (2019).

[37] T. Damour, The Motion of Compact Bodies and Gravitational Radiation, Gen. Relati. Gravit. 89 (1984).

[38] E. E. Flanagan and T. Hinderer, Constraining neutron star tidal Love numbers with gravitational wave detectors, Phys. Rev. D 77, 021502 (2008).

[39] J. Vines, E. E. Flanagan, and T. Hinderer, Post-1-Newtonian tidal effects in the gravitational waveform from binary inspirals, Phys. Rev. D 83, 084051 (2011).

[40] T. Damour, A. Nagar, and L. Villain, Measurability of the tidal polarizability of neutron stars in late-inspiral gravitational-wave signals, Phys. Rev. D 85, 123007 (2012).

[41] S. E. Gralla, On the ambiguity in relativistic tidal deformability, Classical Quant. Grav. 35, 085002 (2018).

[42] E. Poisson, Gravitational waves from inspiraling compact binaries: The quadrupole moment term, Phys. Rev. D 57, 5287 (1998).

[43] A. Bohé, G. Faye, S. Marsat, and E. K. Porter, Quadratic-inspin effects in the orbital dynamics and gravitational-wave energy flux of compact binaries at the $3 \mathrm{PN}$ order, Classical Quant. Grav. 32, 195010 (2015).

[44] L. Baiotti and L. Rezzolla, Binary neutron star mergers: A review of Einstein's richest laboratory, Rep. Prog. Phys. 80, 096901 (2017).

[45] T. Cullen, I. Harry, J. Read, and E. Flynn, Matter effects on LIGO/Virgo searches for gravitational waves from merging neutron stars, Classical Quant. Grav. 34, 245003 (2017).

[46] B. Abbott et al. (LIGO Scientific and Virgo Collaborations), GW170817: Measurements of Neutron Star Radii and Equation of State, Phys. Rev. Lett. 121, 161101 (2018).

[47] B. P. Abbott et al. (LIGO Scientific and Virgo Collaborations), Model comparison from LIGO-Virgo data on GW170817's binary components and consequences for the merger remnant, Classical Quant. Grav. 37, 045006 (2020).

[48] E. K. Porter, Computational resources to filter gravitational wave data with P-approximant templates, Classical Quant. Grav. 19, 4343 (2002).

[49] R. Smith, K. Cannon, C. Hanna, D. Keppel, and I. Mandel, Towards rapid parameter estimation on gravitational waves from compact binaries using interpolated waveforms, Phys. Rev. D 87, 122002 (2013).

[50] P. Canizares, S. E. Field, J. Gair, V. Raymond, R. Smith, and M. Tiglio, Accelerated Gravitational-Wave Parameter Estimation with Reduced Order Modeling, Phys. Rev. Lett. 114, 071104 (2015).

[51] B. Zackay, L. Dai, and T. Venumadhav, Relative binning and fast likelihood evaluation for gravitational wave parameter estimation, arXiv:1806.08792.

[52] S. Droz, D. J. Knapp, E. Poisson, and B. J. Owen, Gravitational waves from inspiraling compact binaries: Validity of the stationary phase approximation to the Fourier transform, Phys. Rev. D 59, 124016 (1999).

[53] A. Buonanno, B. Iyer, E. Ochsner, Y. Pan, and B. Sathyaprakash, Comparison of post-Newtonian templates for compact binary inspiral signals in gravitational-wave detectors, Phys. Rev. D 80, 084043 (2009).

[54] L. Blanchet, B. R. Iyer, C. M. Will, and A. G. Wiseman, Gravitational wave forms from inspiralling compact binaries to second post-Newtonian order, Classical Quant. Grav. 13, 575 (1996).

[55] A. H. Nitz, A. Lundgren, D. A. Brown, E. Ochsner, D. Keppel, and I. W. Harry, Accuracy of gravitational waveform models for observing neutron-star-black-hole binaries in Advanced LIGO, Phys. Rev. D 88, 124039 (2013).

[56] J. Conway and N. Sloane, Sphere Packings, Lattices and Groups, 2nd ed. (Springer-Verlag, New York, 1993).

[57] B. P. Abbott et al. (LIGO Scientific and Virgo Collaborations), Search for post-merger gravitational waves from the remnant of the binary neutron star merger GW170817, Astrophys. J. Lett. 851, L16 (2017).

[58] T. W. Baumgarte, S. L. Shapiro, and M. Shibata, On the maximum mass of differentially rotating neutron stars, Astrophys. J. Lett. 528, L29 (2000).

[59] S. L. Shapiro, Differential rotation in neutron stars: Magnetic braking and viscous damping, Astrophys. J. 544, 397 (2000).

[60] K. Hotokezaka, K. Kiuchi, K. Kyutoku, T. Muranushi, Y.-I. Sekiguchi, M. Shibata, and K. Taniguchi, Remnant massive neutron stars of binary neutron star mergers: Evolution process and gravitational waveform, Phys. Rev. D 88, 044026 (2013).

[61] V. Ravi and P. D. Lasky, The birth of black holes: Neutron star collapse times, gamma-ray bursts and fast radio bursts, Mon. Not. R. Astron. Soc. 441, 2433 (2014).

[62] B. Margalit and B. D. Metzger, Constraining the maximum mass of neutron stars from multi-messenger observations of GW170817, Astrophys. J. Lett. 850, L19 (2017).

[63] A. Bauswein, O. Just, H.-T. Janka, and N. Stergioulas, Neutron-star radius constraints from GW170817 and future detections, Astrophys. J. Lett. 850, L34 (2017).

[64] L. Rezzolla, E. R. Most, and L. R. Weih, Using gravitational-wave observations and quasi-universal relations to constrain the maximum mass of neutron stars, Astrophys. J. Lett. 852, L25 (2018).

[65] D. Radice, A. Perego, F. Zappa, and S. Bernuzzi, GW170817: Joint constraint on the neutron star equation of state from multimessenger observations, Astrophys. J. Lett. 852, L29 (2018).

[66] B. Abbott et al. (LIGO Scientific and Virgo Collaborations), Search for gravitational waves from a long-lived remnant of the binary neutron star merger GW170817, Astrophys. J. 875, 160 (2019).

[67] M. Punturo et al., The third generation of gravitational wave observatories and their science reach, Classical Quant. Grav. 27, 084007 (2010).

[68] B. P. Abbott et al. (LIGO Scientific Collaboration), Exploring the sensitivity of next generation gravitational wave detectors, Classical Quant. Grav. 34, 044001 (2017). 
[69] D. Reitze et al., Cosmic Explorer: The U.S. contribution to gravitational-wave astronomy beyond LIGO, Bull. Am. Astron. Soc. 51, 035 (2019), https://baas.aas.org/pub/ 2020n7i035/release/1.

[70] I. Harry and T. Hinderer, Observing and measuring the neutron-star equation-of-state in spinning binary neutron star systems, Classical Quant. Grav. 35, 145010 (2018).

[71] T. Hinderer, B. D. Lackey, R. N. Lang, and J. S. Read, Tidal deformability of neutron stars with realistic equations of state and their gravitational wave signatures in binary inspiral, Phys. Rev. D 81, 123016 (2010).

[72] See Supplemental Material at http://link.aps.org/ supplemental/10.1103/PhysRevD.104.043008 for animated versions of Figs 1, 2, 4, and 5.

[73] M. Vallisneri, Use and abuse of the Fisher information matrix in the assessment of gravitational-wave parameterestimation prospects, Phys. Rev. D 77, 042001 (2008).

[74] M. Zanolin, S. Vitale, and N. Makris, Application of asymptotic expansions of maximum likelihood estimators errors to gravitational waves from binary mergers: The single interferometer case, Phys. Rev. D 81, 124048 (2010).

[75] S. Vitale and M. Zanolin, Parameter estimation from gravitational waves generated by non-spinning binary black holes with laser interferometers: Beyond the Fisher information, Phys. Rev. D 82, 124065 (2010).

[76] M. Vallisneri, Beyond Fisher: Exact Sampling Distributions of the Maximum-Likelihood Estimator in GravitationalWave Parameter Estimation, Phys. Rev. Lett. 107, 191104 (2011).

[77] S. Vitale and M. Zanolin, Application of asymptotic expansions for maximum likelihood estimators' errors to gravitational waves from binary mergers: The network case, Phys. Rev. D 84, 104020 (2011).

[78] F. Pannarale and F. Ohme, Prospects for joint gravitationalwave and electromagnetic observations of neutron-starblack-hole coalescing binaries, Astrophys. J. Lett. 791, L7 (2014). 